\title{
Lymphocyte Depleted Hodgkin's Lymphoma Presented with Haemolytic Anemia: A Case Report and Literature Review
}

\author{
Ravinder Singh • Soheyl Sheikh • Shambulingappa Pallagatti • \\ Amit Aggarwal - Deepak Gupta - Roopika Handa - Gaurav Goyal • \\ Raman Kumar
}

Received: 29 August 2012/Accepted: 13 April 2013/Published online: 5 May 2013

(C) Association of Oral and Maxillofacial Surgeons of India 2013

\begin{abstract}
Hodgkin's lymphoma accounts for ten per cent of all lymphomas. Lymphomas represent the third most frequent neoplasia on a worldwide scale and constitute $3 \%$ of malignant tumors. In the United States, there are about 8,000 new cases every year. This case report describes a case of lymphocyte-depletion Hodgkin's lymphoma (LDHL) manifested by anemia. LDHL is the least common of the types of Hodgkin's disease, comprising less than $1 \%$ of all cases of this cancer. A 19-year-old Indian male presented with complaint of huge asymptomatic swelling in the right lateral part of neck since 8 months. All the lymph nodes of the right side submandibular, submental, cervical and axillary lymph nodes were rubbery in consistency, tender and were palpable. The $\mathrm{CBC}$ revealed hemoglobin of $7.4 \mathrm{gm} / \mathrm{dL}$ and ESR count was highly raised up to $125 \mathrm{~mm} /$ first hour. Direct Coomb's test detected multiple antibodies IgG type, which was consistent with the diagnosis of Haemolytic anemia. Ultrasonography of the neck showed enlarged discrete lymph nodes on the right side of the neck. For further evaluation patient was subject for ultrasonography of the abdomen which revealed there was of enlargement of both spleen and liver. Lymph node FNAC revealed classic LDHL. Anemia and swelling resolved after completion of chemotherapy with adriamycin, bleomycin, vinblastine, and dacarbazine (ABVD) after 6 cycles. The literature review and our case report discuss the optimal management of this Hodgkin's lymphoma. To our knowledge, this is the first case of LDHL with Haemolytic Anemia treated with only ABVD regime.
\end{abstract}

\footnotetext{
R. Singh $\cdot$ S. Sheikh $\cdot$ S. Pallagatti $\cdot$ A. Aggarwal $\cdot$ D. Gupta $\cdot$ R. Handa $\cdot$ G. Goyal $(\bowtie) \cdot$ R. Kumar Department of Oral Medicine and Radiology, M.M. College of Dental Sciences and Research, Mullana, Ambala 133203, Haryana, India e-mail:dr.gaurav867@gmail.com
}

Keywords Hodgkin's lymphoma - Lymph nodes · Swelling · Anemia $\cdot$ Ultrasonography $\cdot$ ABVD

\section{Introduction}

Lymphomas represent the third most frequent neoplasia on a worldwide scale and constitute $3 \%$ of malignant tumors $[1,2]$.Their prevalence progressively grows at the annual rate of 3. The WHO classification of tumors, which result from the hematopoietic tissues and the lymphoid tissues, allows to distinguish lymphoid neoplasias, according to the cell line and the differentiation into:

- Hodgkin's lymphoma

- B cell neoplasia

- Peripheral B cell neoplasia

- B cell proliferations of uncertain malignant potential

- T cell neoplasia

- Peripheral T/NK-cell neoplasia

- $\mathrm{T}$ cell proliferations of uncertain malignant potential [1-3]

Hodgkin's lymphoma (HL) is a solid tumor that arises from B lymphocytes. It was first described in 1832 [4, 5]. Since that time, this neoplasm has been extensively studied; currently, it is classified into classic Hodgkin lymphoma (cHL) and lymphocyte predominant variant. It frequently represents a challenge for diagnosis when presents as a mediastinal mass. cHL is further divided into four categories-nodular sclerosis, mixed cellularity, lymphocyte rich, and lymphocyte depleted. Other lymphomas, especially primary mediastinal large B cell lymphoma, show borderline histological features similar to cHL $[5,6]$.

In the developed countries, HL roughly accounts for $10 \%$ of all diagnosed lymphomas. This neoplasm follows a 
bimodal age distribution having a peak in early adulthood and then in the seventh decade [5]. It has male predominance. Worldwide, nodular sclerosis subtype accounts for majority of all cHL, whereas lymphocytic depletion type accounts for less than $1 \%$ [5].

\section{Incidence}

\begin{tabular}{lll}
\hline Race/ethnicity & Male & Female \\
\hline All races & 3.2 per 100,000 & $\begin{array}{c}2.5 \text { per } 100,000 \\
\text { women }\end{array}$ \\
& men & 2.4 per 100,000 \\
Black & 3.1 per 100,000 & women \\
& men & 2.7 per 100,000 \\
White & 3.3 per 100,000 & women \\
& men & 1.1 per 100,000 \\
Asian/Pacific & 1.5 per 100,000 & women \\
Islander & men & \\
\hline
\end{tabular}

In classical presentations, cervical lymph nodes are involved in approximately three quarters of cases at diagnosis. Hodgkin's lymphoma is a disease affecting primarily lymph nodes with secondary extranodal spread. It accounts for 1-5\% of head and neck tumors [7]. The differential diagnosis between a non-Hodgkin lymphoma of the head and neck region and Hodgkin's disease can be difficult. Some cases reported in the literature to be Hodgkin's disease of the Waldeyer's ring probably represent non-Hodgkin's lymphomas [7, 8]. The main histopathologic feature of Hodgkin's lymphoma is the presence of Reed-Stenberg cells (binucleated or multinucleated, with big and clear nuclei and intensely colored "owl's eyes" nucleoli). The above-mentioned ReedStenberg cells are not found in the so-called non-Hodgkin's lymphoma, a category that includes all the other histopathologic entities that are not related to Hodgkin's disease [9].

From a clinical point of view and in almost all cases, Hodgkin's lymphoma is a nodular lesion that rarely involves the extranodal areas, whereas NHL frequently has an extranodal onset. In addition, whereas Hodgkin's disease spreads in the nodal groups in a contiguous fashion, in NHL the nodal evolution proceeds randomly. This means that it does not proceed in a contiguous fashion, but is instead unforeseeable (obviously, this aspect has important repercussions on the therapeutic protocol) [2].

From 2005-2009, the median age at death for Hodgkin lymphoma was 64 years of age. Approximately $1.6 \%$ died under age of 20 years; $12.9 \%$ between 20 and 34 years; $10.5 \%$ between 35 and 44 years; $11.8 \%$ between 45 and 54 years; $14.5 \%$ between 55 and 64 years; $16.4 \%$ between 65 and 74 years; $22.3 \%$ between 75 and 84 years; and $10.0 \% 85+$ years of age. The age-adjusted death rate was 0.4 per 100,000 men and women per year. These rates are based on patients who died in 2005-2009 in the US.

Epstein-Barr virus (EBV) or its remnants have been identified in Sternberg-Reed cells. This supports the hypothesis that EBV is associated with HL. However, it is not clear whether its presence is necessary for oncogenesis, since 40-60\% of HL is EBV negative [10]. According to Dinand et al. [11] in 2006, EBV detection in $96.6 \%$ of childhood HL in a population with almost universal EBV seroconversion, and in none of the control lymph nodes, suggests a causative role of EBV in most cases of Indian childhood HL.

Autoimmune hemolytic anemia (AIHA) is rarely seen in Hodgkin lymphoma (HL) patients, with a reported incidence of $0.2-4.2 \%$. Sporadic case reports and reviews have shown that when AIHA occurs in HL patients, it happens mostly at stages III and IV of nodular sclerosis HL (NSHL) or mixed cellularity HL (MCHL) [12]. The mechanism of anemia varies; it could be secondary to chronic disease reflecting the underlying malignant process. In advanced disease anemia occurs from bone marrow infiltration by malignant cells [5]. We present a case of hemolytic anemia at the time of diagnosis of classic HL, with a detailed review of the literature of HL accompanied by HA. Our goal is to give clinicians an overview of this complication for better management in the future.

\section{Case Report}

A 19 year old boy reported to the department of oral medicine and radiology with the complaint of big asymptomatic swelling in the right lateral part of neck since 8 months. The swelling was insidious in onset, gradually increasing in size and attained the present size. The patient gave a history of missing tooth in the same region. There was no history of trauma. Mouth opening was normal with no deviation on maximum opening. The patient's medical, surgical and dental histories were not significant.

On physical examination there was a single, large, diffuse swelling measuring approximately $20 \times 18 \mathrm{~cm}$ present on the right side of neck which further caused facial asymmetry. Swelling was elliptical in shape, nearly approaching the midline (Fig. 1). It extended from midline of neck to below the occipital region mesiodistally and from below the auricular region to supraclavicular region anteroposteriorly (Fig. 2). The colour of swelling was same as that of surrounding skin. On palpation, swelling was non tender, firm in consistency and smooth in texture. The temperature of swelling was raised as compared to that of surrounding skin. Swelling was not fixed to underlying structures. It was slightly movable in vertical and horizontal direction. Swelling was non fluctuant, noncompressible but pulsatile in 
nature. All the lymph nodes of the right side-submandibular, submental, cervical and axillary lymph nodes were rubbery in consistency, tender and were palpable.

The CBC revealed increased polymorphs $85 \mathrm{HPF}$ and decreased lymphocytes $13 \mathrm{HPF}$, hemoglobin $7.4 \mathrm{gm} / \mathrm{dL}$, and platelets count was $225,000 \mathrm{gm} / \mathrm{dL}$. ESR count was highly raised up to $125 \mathrm{~mm} /$ first hour and serum lacticodehydrogenase was in normal limits. Chemistry profile was within normal limits.

$\mathrm{X}$-ray examination of the chest revealed no lung lesions and normal cardiac size. After that the patient was subjected for ultrasonography of the neck, which revealed there were enlarged discrete hypoechoic lymph nodes on the right side of the neck with the loss of hilum (Fig. 3). For further evaluation patient was subject for ultrasonography of the abdomen which revealed there was enlarged spleen with multiple scattered hypoechoic areas, as well as liver was also enlarged without any focal lesion which was further suggestive of hepatosplenomegaly (Fig. 4). Based on ultrasonographic evalution, the diagnosis was made as lymphoma of the right side of neck.

FNAC of the lesion showed numerous atypical cells scattered singly. Cells showed marked nuclear pleomorphism, binucleated and multinucleated forms (Reed Stenberg cells) along with multiple mitotic figures (Fig. 5). FNAC results were consistent with the clinical and radiographic diagnosis of lymphocytic depletion Hodgkin's lymphoma. Direct Coomb's test was positive, consistent with the diagnosis of HA. These antibodies were further identified as being IgG.

After the confirmation of final diagnosis, patient was referred to department of general surgery. Urgent blood transfusion was ordered, as the patient was anemic and his hemoglobin level was $7.4 \mathrm{gm} / \mathrm{dl}$. As the hemoglobin level was achieved to optimum level, chemotherapy (ABVD) regime was started. ABVD regimen comprised of drugs named doxorubicin, bleomycin, vinblastine, and dacarbazine. Combined modality

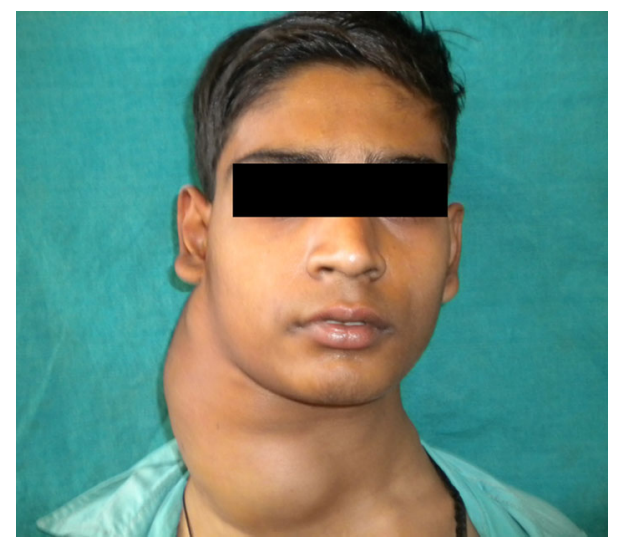

Fig. 1 A large, diffuse swelling measuring approximately $20 \times 18 \mathrm{~cm}$ present on the right side of neck

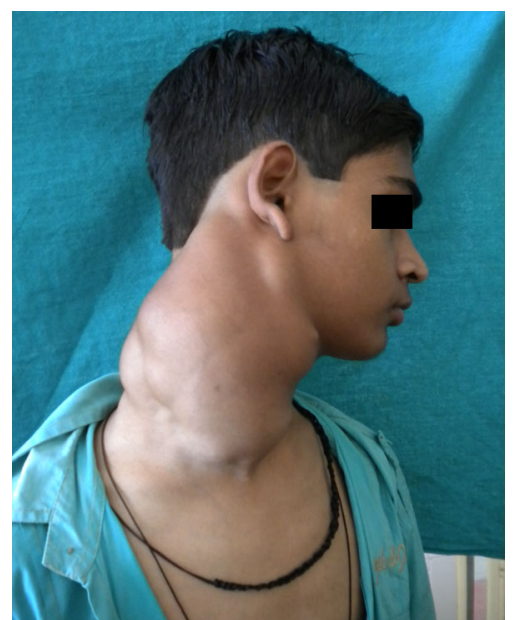

Fig. 2 Lateral profile of patient showing the extension of the swelling

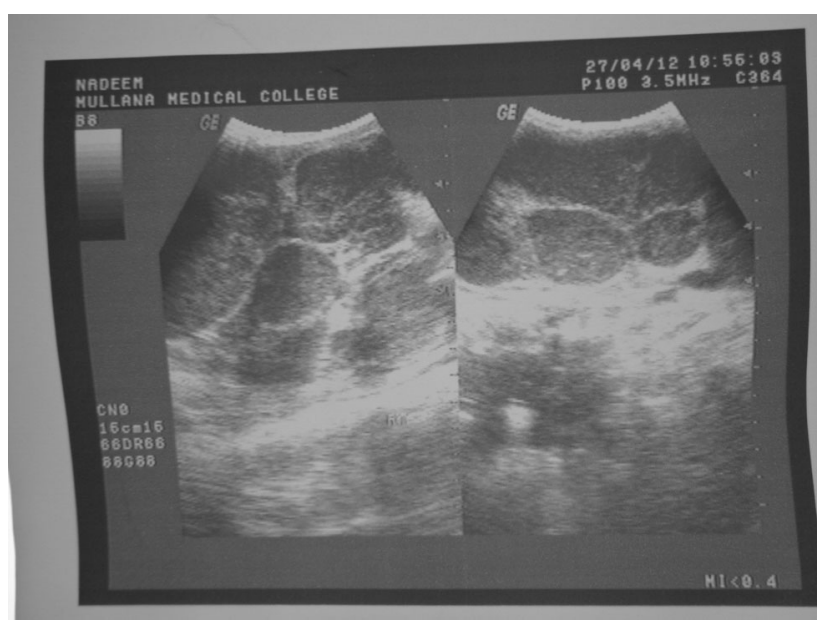

Fig. 3 Ultrasonography of the neck showing enlarged discrete hypoechoic lymph nodes on the right side of the neck

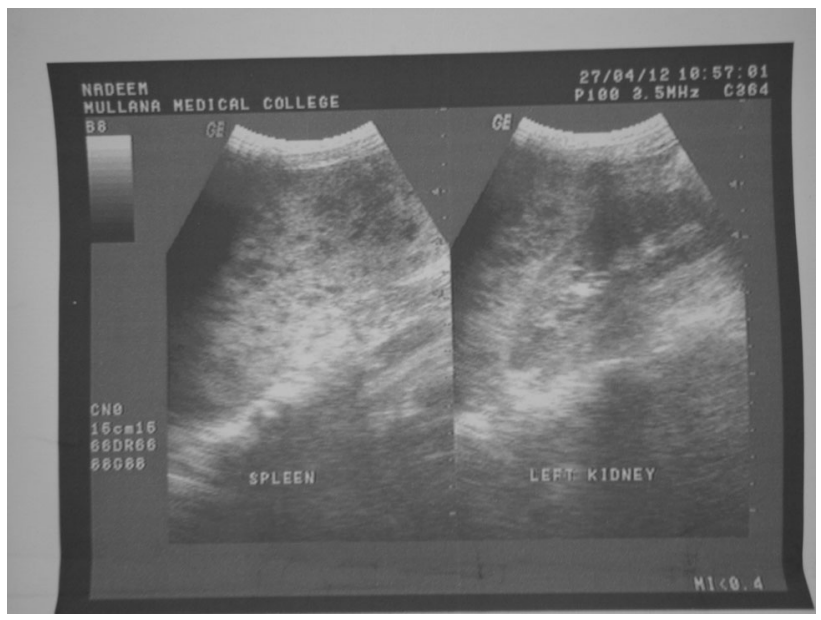

Fig. 4 Ultrasonography of the abdomen 


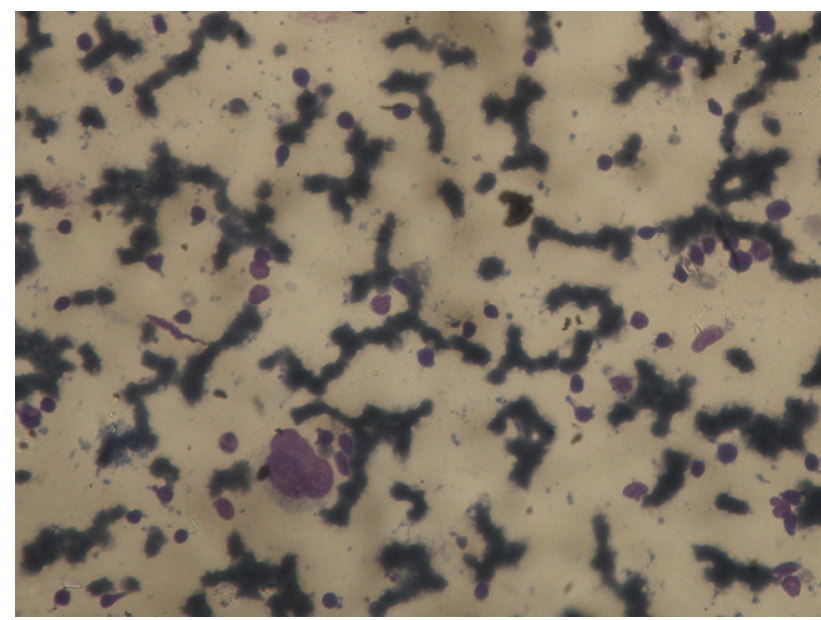

Fig. 5 FNAC of the lesion showed numerous atypical cells scattered singly. Cells showed marked nuclear pleomorphism, binucleated and multinucleated forms (Reed Stenberg cells) along with multiple mitotic figures

consisting of doxorubicin $50 \mathrm{mg} / \mathrm{m}^{2}$ IV plus bleomycin $15 \mathrm{IU} /$ $\mathrm{m}^{2} \mathrm{IV}$ plus vinblastine $10 \mathrm{mg} / \mathrm{m}^{2}$ IV plus dacarbazine $400 \mathrm{mg} / \mathrm{m}^{2}$ IV on days 1 and 15 . ABVD was done in cycles. Each cycle consisted of giving the patient injections of these 4 drugs twice (on days 1 and 15). Cycles were repeated in 4 week intervals, that mean the second cycle starts 2 weeks after day 15 of the first cycle (on day 29), and so on. A total of 6 cycles of the $\mathrm{ABVD}$ regime was given to the patient.

After receiving first cycle of chemotherapy the patient was discharged with advice to follow-up for further chemotherapy. At each visit of the chemotherapy regime, patient was examined carefully and considerable decrease in size of lesion was observed. After 4 cycles of ABVD, lesion showed $90 \%$ reduction and a complete regression of cervical lymphadenopathies (Fig. 6). Repeated blood examination showed improved hemolytic anemia. An Ig test showed increased IgG level. This response was maintained after 6 cycles of ABVD. A complete remission was confirmed after the end of treatment (Fig. 7). A regular 1 year follow-up was done. The patient was disease free, without any sign and symptoms. The patient can now be designated as disease free after a regular follow-up of 1 year. It indicated a good prognosis.

\section{Discussion}

Lymphocyte-depleted Hodgkin's lymphoma is a type of Hodgkin's disease, a cancer of lymph cells. It is the least common of the types of Hodgkin's disease, comprising only $<1 \%$ of all cases of this cancer. It is more common in the less industrialized part of the world. Lymphocyte-depleted Hodgkin's is more common in older people. It is also more common in patients with Acquired Immunodeficiency

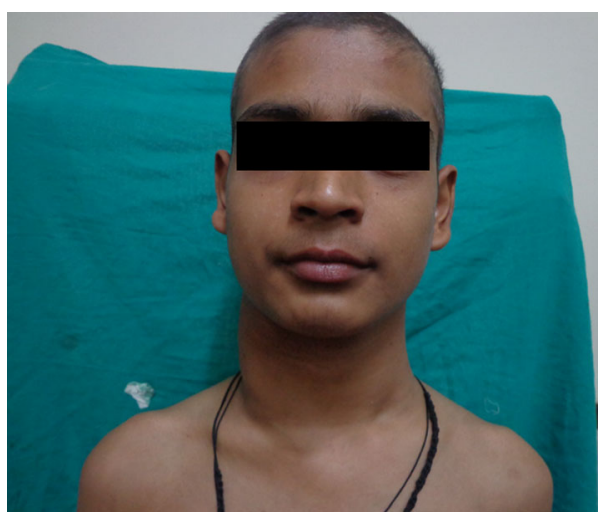

Fig. 6 After 4 cycles of ABVD, lesion showed $90 \%$ reduction and a complete regression of cervical lymphadenopathies
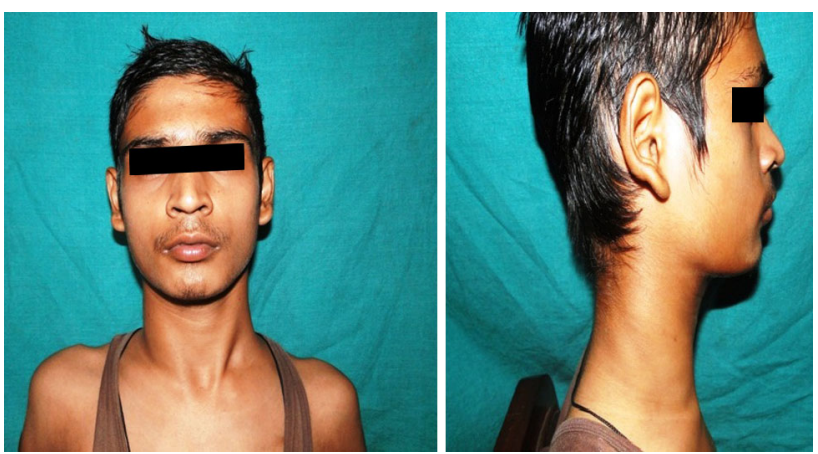

Fig. 7 Post-operative photograph of the patient after the 6 cycles of chemotherapy

Syndrome (AIDS) who develop Hodgkin's lymphoma. On the contrary, our patient was a healthy young male aged 19 years.

In lymphocyte-depleted type, majority of patients are diagnosed at an advanced stage, with lymphoma commonly affecting the liver, spleen and bone marrow, as seen in our case, in which patient had hepatosplenomegaly.

In most of the cases, Classic Hodgkin lymphoma presents with symptoms like night sweat, fever, and weight loss which are encountered in about twenty per cent of patients. Pel-Ebstein fever, although less common, is also associated with this illness. It usually happens at irregular intervals of several days or weeks before it finally disappears. Seldoms symptoms such as pruritus, which may precede the diagnosis of cHL for months, cholestatic liver disease, skin lesions such as ichthyosis, acrokeratosis, and hyperpigmentation, neurological symptoms secondary to central nerve system malignant cells infiltration, and nephrotic syndromes resulting from hypersecretion of toxic lymphokines can be observed at onset of clinical disease. In our case, patient reported with swelling only, no other symptoms were associated with it $[5,13,14]$.

Patients with Hodgkin's disease of the lymphocytedepleted subtype (LDHL) are said to have a poor prognosis. However, reports of this subtype are complicated by 
the fact that the histologic diagnosis of LDHL is often not straightforward, and its distinction from aggressive nonHodgkin's lymphomas (NHL) can be difficult. Kant et al. [15] reviewed the patients with LDHL at the National Cancer Institute (NCI) in light of an additional decade of experience with neoplastic and non-neoplastic conditions mimicking Hodgkin's disease. Of 198 patients who received MOPP (mechlorethamine, vincristine, procarbazine, prednisone) treatment at the NCI for Hodgkin's disease between 1964 and 1976, 43 (22\%) were originally classified as LDHL. The initial diagnostic biopsies from 39 of these patients were reviewed and revealed ten with NHL, nine with LDHL, and 13 with nodular sclerosing Hodgkin's disease of the lymphocyte-depleted subtype (NSLD). The other seven patients had Hodgkin's disease without a lymphocyte-depleted component. The pathologic review was done without knowledge of clinical features which were examined after review in the three major subgroups. Complete remissions were attained by 67 and $85 \%$ of patients in the LDHD and NSLD groups, respectively: median survival had not been reached in either group with a median of 14 years of follow-up [15].

The relationship of autoimmune hemolytic anemia with $\mathrm{cHL}$ has been recognized for more than 40 years. However, there is no extensive primary medical literature that has characterized this association. It has not been as extensively studied as in the case of diffuse large B cell lymphoma [13, 14]. According to Lechner and Chen [13] 34 cases of AIHA associated with cHL have been reported in PubMed from 1951 to 2009, in none of these cases AIHA was associated with LRHL and LDHL; eighteen of the cases were mixed cellularity, eleven were nodular sclerosis, and eight were nonclassified. The time of presentation of AIHA varies. Interestingly, in eight of these cases its onset preceded the diagnosis of HL; time interval ranged from 5 months to 21 years. In six of these cases, AIHA appeared as a first sign of relapse. Additionally, in two cases, AIHA presented years after satisfactory treatment, and both patients where in complete remission. In majority of cases, the culprit antibody is $\mathrm{IgG}$, as seen in our case report. However, there have been cases of IgA-mediated AIHA in patients with HL [5, 13]. Some researchers have suggested that AIHA results from a paraneoplastic syndrome that develops due to the aberrant production of hormones, cytokines, or antibodies by neoplastic cells. It has been also hypothesized that these antibodies cross-react with red blood cells promoting hemolysis. This assumption is based on the clinical observation that AIHA respond well to chemotherapy and steroids, readily resolving shortly after treatment is started [13, 14]. As seen in our case, when the patient was in 6th cycle of chemotherapy, the $\mathrm{Hb}$ level increased to $12 \mathrm{gm} / \mathrm{dl}$ and increased the IgG level also increased.
The treatment ofl LDHD should be a sequential combination of chemotherapy and radiotherapy.

Literature suggest that 6 cycles of a chemotherapeutic regimen, doxorubicin, bleomycin, vinblastine, dacarbazine [ABVD] polychemotherapy is the appropriate standard regimen and involved field radiotherapy with target volume given as an intermediate dosage (25-40 Gy) targeting the Waldeyer's ring and cervical lymph nodes should be the first line of treatment for patients with HL. However, some authors concluded that chemotherapy alone with ABVD can be an option in the treatment of nonbulky Hodkin disease [16]. If the long-term toxic risks of radiation are to be avoided, then this approach should be the treatment of choice, especially for patients younger than 40 years. There have been a host of long-term follow-up studies that have clearly defined the carcinogenic and cardiotoxic risks of curative radiation therapy. The projected risk of one or both of these complications approaches $40 \%$ at 25-30 years, and is especially true for children and adolescents $[16,17]$. Patients with mediastinal tumors will receive radiation to some cardiac and lung parenchymal tissue. There is recent studies of ABVD alone, from a Memorial Sloan-Kettering randomized trial with 152 patients, of whom 76 received ABVD alone, and 76, combined ABVD and irradiation [12]. At 60 months, there were no differences in FFP or overall survival [18]. The ABVD arm had a complete remission rate of $94 \%$, progression-free survival of $81 \%$ and an overall survival of $90 \%$ [18]. So with these type of articles in mind, in our case report, only chemotherapy was given to the patient and a satisfactory result was achieved. To our knowledge, this is the first case of LDHL with Hemolytic Anemia treated with ABVD.

Till 2012, 11 deaths had been reported. Six patients died directly from HL, with a median age of 24 years, who died of various causes, including congestive heart failure, secondary to long-standing AIHA with HL stage, metastatic carcinoma of the biliary duct with HL, and brain toxoplasmosis after months of steroid treatment. Note that 9 of the 11 patients died before 1974, and 2 died in 1995-1996, suggesting that more effective chemotherapy, such as ABVD, might have improved survival rates [12].

\section{Conclusion}

We describe a case of LDHL that presented with anemia that resolved after successful treatment of the disease. Review of the literature revealed only very less reported cases of cHL of this subtype. Although infrequent as the first manifestation of cHL, clinicians should consider the differential diagnosis of this malignancy in patients affected by seemingly hemolytic anemia. Further studies are 
necessary to precisely define the exact pathophysiology of this entity.

Rapid diagnostic assessments, together with an adequate histopathologic verification, are indeed essential to improve the management and the prognosis of this disease.

\section{References}

1. Lu P (2005) Staging and classification of lymphoma. Semin Nucl Med 35:160-164

2. Inchingolo F, Tatullo M, Abenavoli FM, Marrelli M, Inchingolo AD, Inchingolo AM, Dipalma G (2011) Non-Hodgkin lymphoma affecting the tongue: unusual intra-oral location. Head Neck Oncol 3(1):1-5. doi: 10.1186/1758-3284-3-1

3. Jaffe ES, Harris NL, Stein H (2002) World Health Organization classification of tumours, pathology and genetics, tumours of haematopoietic and lymphoid tissues. Ann Oncol 13:490-491

4. Buglio D, Younes A (2010) Histone deacetylase inhibitors in Hodgkin lymphoma. Invest New Drugs 28(suppl 1):S21-S27. doi:10.1007/s10637-010-9588

5. Hurtado-Cordovi JM, Verma V, Gotlieb V, Frieri M (2011) Lymphocyte rich Hodgkin's lymphoma presented with warm hemolytic anemia: a case report and literature review. Case Rep Hematol: 385408. doi: 10.1155/2011/385408

6. Banerjee D (2011) Recent advances in the pathobiology of Hodgkin's lymphoma: potential impact on diagnostic, predictive, and therapeutic strategies. Adv Hematol 439456:11-26. doi:10.1155/ 2011/439456

7. Malis DD, Moffat D, McGarry GW (1998) Isolated nasopharyngeal Hodgkin's disease presenting as nasal obstruction. Int $\mathbf{J}$ Clin Pract 52:343-346

8. Aloulou S, Farhat H, Bosq J, Vanel D, Ribrag V, Turhan AG, Girinsky T, Fermé C (2002) Hodgkin's disease primarily involving the oropharynx: case report and review of the literature. Hematol J 3:164-167

9. Whitt JC, Dunlap CL, Martin KF (2007) Oral Hodgkin lymphoma: a wolf in wolf's clothing. Oral Surg Oral Med Oral Pathol Oral Radiol Endod 104:e45-e51

10. Kapadia SB, Roman LN, Kingma DW, Jafe ES, Frizerra G (1995) Hodgkin's disease of Waldeyer's ring: clinical and immunophenotypic findings and association with Epstein-Barr virus in 16 cases. Am J Surg Pathol 19:1431-1439

11. Dinand V, Dawar R, Arya LS et al (2007) Hodgkin's lymphoma in Indian children: prevalence and significance of Epstein-Barr virus detection in Hodgkin's and Reed-Sternberg cells. Eur J Cancer 43:161-168. doi:10.1016/j.ejca.2006.08.036

12. Feng Q, Zak D, Daya R (2012) Autoimmune hemolytic anemia and classical Hodgkin lymphoma: a case report and literature review. Clin Adv Hematol Oncol 10:270-277

13. Lechner K, Chen YA (2010) Paraneoplastic autoimmune cytopenias in Hodgkin lymphoma. Leuk Lymphoma 51:469-474

14. Siddiqui N, Aleem A (2009) Autoimmune haemolytic anaemia preceding the diagnosis of Hodgkin's disease: a report of two cases and review of the literature. J Park Med Assoc 59:316-318

15. Kant JA, Hubbard SM, Longo DL, Simon RM, DeVita VT Jr, Jaffe ES (1986) The pathologic and clinical heterogeneity of lymphocyte-depleted Hodgkin's disease. J Clin Oncol 4:284-294

16. Canello George P (2005) Chemotherapy alone for early Hodgkin's lymphoma: an emerging option. J Clin Oncol 23:4574-4576

17. van Leeuwen FE, Klokman WJ, Veer MB, Hagenbeek A, Krol $\mathrm{AD}$, Vetter U, Schaapveld M, van Heerde P, Burgers JM, Somers R, Aleman BM (2000) Long-term risk of second malignancy in survivors of Hodgkin's disease treated during adolescence or young adulthood. J Clin Oncol 18:487-497

18. Straus DJ, Portlock CS, Qin J, Myers J, Zelenetz AD, Moskowitz C, Noy A, Goy A, Yahalom J (2004) Results of a prospective randomized clinical trial of doxorubicin, bleomycin, vinblastine, and dacarbazine (ABVD) followed by radiation therapy (RT) versus $A B V D$ alone for stages I, II, and IIIA nonbulky Hodgkin disease. Blood 104:3483-3489 